PROCEEDINGS OF THE

AMERICAN MATHEMATICAL SOCIETY

Volume 103, Number 1, May 1988

\title{
SCALAR-REFLEXIVE RINGS
}

\author{
DON HADWIN AND JEANNE WALD KERR
}

(Communicated by Donald Passman)

\begin{abstract}
A module over a commutative ring is scalar-reflexive if the only endormorphisms that leave invariant every submodule are scalars. We investigate the class of rings for which every module is scalar-reflexive and the class of rings for which every finitely generated module is scalar-reflexive. For a certain class of rings we show that these properties are equivalent to every finitely generated module being a direct sum of cyclic modules.
\end{abstract}

It is an elementary exercise in linear algebra to show that if $T$ is a linear transformation on a vector space that leaves invariant every linear subspace (i.e., every vector is an eigenvector), then $T$ must be a scalar. This paper begins an investigation of the analogous question for modules over commutative rings.

If $R$ is a commutative ring with 1 , call a unitary $R$-module $M$ scalar-reflexive if each $T$ in $\operatorname{End}_{R}(M)$ that leaves invariant every $R$-submodule is a scalar (i.e., an $R$-multiple of the identity endomorphism). The problem of determining whether a given $R$-module $M$ is scalar-reflexive is highly nontrivial. Consider the example in which $R$ is a commutative algebra of linear transformations on a vector space $M$ over a field $F$. The question of $M$ being a scalar-reflexive $R$-module translates into the question of whether a linear transformation $T$ must be in $R$ if it commutes with every element of $R$ (i.e., $T \in \operatorname{End}_{R}(M)$ ) and leaves invariant all of the linear subspaces that are invariant for every element of $R$ (i.e., the $R$-submodules of $M$ ). This problem in linear algebra seems hopeless even when $M$ is finite-dimensional (see [1]).

It was shown by L. Brickman and P. A. Fillmore [2] that if $F$ is an algebraically closed field, then every finite-dimensional $F[x]$-module is scalar-reflexive. This result was extended to arbitrary fields in [3], where it was also shown that if $F$ is an infinite field, then the only $F[x]$-modules that are not scalar-reflexive are torsion and faithful, i.e., where $x$ acts as a locally algebraic transformation that is not algebraic.

The purpose of this paper is to study two classes of commutative rings. Call a commutative ring $R$ with identity strongly scalar-reflexive if every $R$-module is scalar-reflexive, and call $R$ strictly scalar-reflexive if every finitely generated $R$ module is scalar-reflexive. The adverb "strictly" is motivated by the strict topology on $\operatorname{End}_{R}(M)$. A subset $U$ of $\operatorname{End}_{R}(M)$ is strictly open if, for each $T$ in $U$, there is a finite subset $E$ of $M$ such that $\left\{S \in \operatorname{End}_{R}(M):\left.S\right|_{E}=\left.T\right|_{E}\right\} \subset U$. Equivalently,

Received by the editors March 18, 1986 and, in revised form, March 5, 1987. Presented at the special session on ring theory and invariant theory at the summer meeting of the AMS on August 7, 1987.

1980 Mathematics Subject Classification (1985 Revision). Primary 13C13, 13E10, 13E15, 13F10; Secondary 13A10, 15A04. 
a net $\left\{T_{n}\right\}$ in $\operatorname{End}_{R}(M)$ converges strictly to $T$ if, for each finite subset $E$ of $M$, there is an index $N$ such that $n>N$ implies that $\left.T_{n}\right|_{E}=\left.T\right|_{E}$. Since the set of endomorphisms leaving invariant every submodule is strictly closed, it follows that an $R$-module $M$ can be scalar-reflexive only if the set of scalars is strictly closed in $\operatorname{End}_{R}(M)$. It is an elementary exercise to show that a commutative ring $R$ is strictly scalar-reflexive if and only if, for every $R$-module $M$, the set of $R$-endomorphisms that leave invariant every $R$-submodule is the strict closure of the set of scalars. It is thereby clear that a ring $R$ is strongly scalar-reflexive if and only if it is strictly scalar-reflexive and, for every $R$-module $M$, the set of scalars is strictly closed in $\operatorname{End}_{R}(M)$.

Obviously, the classes of strictly and strongly scalar-reflexive rings are each closed under homomorphic images and finite direct sums. Hence, finite direct sums of fields are strongly scalar-reflexive; these are the only semisimple strongly scalar-reflexive rings.

THEOREM 1. Suppose $R$ is a semisimple commutative ring with identity. The following are equivalent.

(1) $R$ is strongly scalar-reflexive,

(2) $R$ is a finite direct sum of fields,

(3) for every $R$-module $M$, the set of scalars is strictly closed in $\operatorname{End}_{R}(M)$.

PROOF. The implications $(2) \Rightarrow(1) \Rightarrow(3)$ are clear. We now prove that $(3) \Rightarrow(2)$. Let $M$ and $P$, respectively, be the direct sum and product of all of the rings of the form $R / \mathscr{I}$, with $\mathscr{I}$ a maximal ideal in $R$. Let $\pi: R \rightarrow P$ be the product of the natural quotient maps from $R$ into the $R / \mathscr{I}$ 's. Since $R$ is semisimple, the map $\pi$ must be injective.

Since $M$ is an ideal in $P$, the elements of $P$, acting by multiplication, can be viewed as $R$-endomorphisms on $M$. Moreover, it follows from the Chinese remainder theorem that $P$ is contained in the strict closure of the scalars on $M$. Thus, by (3), the map $\pi$ is onto, which implies that $\pi$ is a ring isomorphism. However, $M$ is an ideal in $P$ and $\pi^{-1}(M)$ is not contained in any maximal ideal in $R$. Thus $M=P$, which implies that the direct product $P$ contains finitely many factors. Hence (2) is true.

The above theorem shows why we were motivated to study the class of strictly scalar-reflexive rings. Even nice rings like $\mathbf{Z}$ and $F[x]$ are not strongly scalarreflexive; however, we shall soon see that these rings are strictly scalar-reflexive.

Clearly, every cyclic $R$-module is scalar-reflexive. While the proof of Theorem 1 shows that arbitrary direct sums of cyclic modules need not be scalar-reflexive, certain restricted direct sums are.

LEMMA 2. Suppose $R$ is a commutative ring with identity and the $R$-module $M$ is the direct sum of a family $\left\{R x_{k}: k \in K\right\}$ of cyclic submodules. For each $k$ in $K$, let $\operatorname{Ann}\left(x_{k}\right)=\left\{r \in R: r x_{k}=0\right\}$, and suppose that there is a finite subset $E$ of $K$ such that $\bigcap\left\{\operatorname{Ann}\left(x_{k}\right): k \in E\right\}=\bigcap\left\{\operatorname{Ann}\left(x_{k}\right): k \in K\right\}$. Then $M$ is scalar-reflexive.

ProOF. Suppose that $T \in \operatorname{End}_{R}(M)$. It suffices to show that there is an $r$ in $R$ such that $T x_{k}=r x_{k}$ for every $k$ in $K$. First let $x=\sum_{k \in E} x_{k}$. Since $T$ leaves invariant the submodule $R x$, we know that there is an $r$ in $R$ such that $T x=r x$. Similarly, for each $k$ in $E$, there is an $r_{k}$ in $R$ such that $T x_{k}=r_{k} x_{k}$. It follows that $T x_{k}=r x_{k}$ for each $k$ in $E$. 
Next suppose that $m \in K \backslash E$. Applying the preceding argument to $y=x_{m}+x$, we see that there is an $s$ in $R$ such that $T x_{m}=s x_{m}$ and $T x=s x$. Since $T x=r x$, it follows that $r-s \in \bigcap\left\{\operatorname{Ann}\left(x_{k}\right): k \in E\right\} \subset \operatorname{Ann}\left(x_{m}\right)$. Thus $T x_{m}=s x_{m}=r x_{m}$. Thus $T x_{k}=r x_{k}$ for every $k$ in $K$.

While many nice PID's are not strongly scalar-reflexive, the following proposition shows how the preceding lemma implies that any nontrivial homomorphic image of a PID is strongly scalar-reflexive.

PROPOSITION 3. If $R$ is a PID and $r$ is a nonzero nonunit, then $R / r R$ is strongly scalar-reflexive.

PROOF. It follows from [6] that every module over $R / r R$ is a direct sum of cyclic modules. Moreover, $R$ is a UFD; thus $R / r R$ has only finitely many ideals. The proof now follows from Lemma 2.

Note that Lemma 2 implies that a finite direct sum of cyclic modules is scalarreflexive. Thus if $R$ is an FGC ring [8] (i.e., every finitely generated $R$-module is a direct sum of cyclic modules), then $R$ is strictly scalar-reflexive.

PrOPOSITION 4. Every commutative FGC ring with identity is strictly scalarreflexive.

Since it is often easy to show that a ring is not strictly scalar-reflexive, the preceding result can be used to show that a ring is not an FGC ring (e.g., Lemma 7 and later results).

The following corollary extends the Brickman-Fillmore result [2] and its extension in [3].

COROLlARY 5. If $T$ is a linear transformation on a vector space $V$ over a field $F$, and if $S$ is a linear transformation on $V$ such that $S T=T S$ and $S$ leaves invariant every $T$-invariant subspace of $V$, then $S$ is in the strict closure of $\{p(T): p \in F[x]\}$.

Proof. Consider $V$ as an $F[x]$-module by $p v=p(T) v$ for every $p$ in $F[x]$ and every $v$ in $V$. The result follows from Proposition 4 and the fact that $F[x]$, being a PID, is an FGC ring.

Since strongly and strictly scalar-reflexive rings must have an identity, these classes of rings are not closed under infinite direct sums. However, with the suitable adjunction of an identity, the class of strictly scalar-reflexive rings is closed under arbitrary direct sums.

THEOREM 6. Suppose that $R$ is a strictly scalar-reflexive ring and $\left\{A_{k}: k \in K\right\}$ is a family of strictly scalar-reflexive $R$-algebras. Then the $R$-subalgebra of $\prod_{k} A_{k}$ generated by $\sum_{k} A_{k}$ and the identity in $\prod_{k} A_{k}$ is strictly scalar-reflexive.

Proof. Let $S$ denote the direct sum of the $A_{k}$ 's, and let $A$ denote the $R$-algebra generated by $S$ and the identity $e$ in the direct product of the $A_{k}$ 's. Let $M$ be a finitely generated $A$-module with generators $x_{1}, \ldots, x_{n}$, i.e., $M=A x_{1}+\cdots+A x_{n}$. Let $M_{0}=S x_{1}+\cdots+S x_{n}$. Suppose that $T \in \operatorname{End}_{A}(M)$ and $T$ leaves invariant every $A$-submodule of $M$. Then $T\left(M_{0}\right) \subset M_{0}$, and $T$ induces an $A$-endomorphism $\hat{T}$ on $\hat{M}=M / M_{0}$ defined by $\hat{T}\left(x+M_{0}\right)=T x+M_{0}$. Clearly, $\hat{T}$ leaves invariant every submodule of $\hat{M}$. Moreover, since $S$ annihilates $\hat{M}$, it follows that $\hat{M}$ is a finitely 
generated $R$-module (where $R$ acts on $\hat{M}$ as $R e$ ) and that $\hat{T}$ is an $R$-endomorphism that leaves invariant every $R$-submodule of $\hat{M}$. Since $R$ is strictly scalar-reflexive, we know that there is an $r$ in $R$ such that $\hat{T} y=r y$ for every $y$ in $\hat{M}$. Thus, for each $x$ in $M,(T-r) x \in M_{0}$, which implies that there is an idempotent $p$ in $S$ ( $p$ is the sum of the identity elements of finitely many of the $A_{k}$ 's) such that $(T-r) x=p(T-r) x$. However, for each $x$ in $M$, there is an $a$ in $A$ such that $(T-r) x=a x$, since $A x$ is an $A$-submodule of $M$. Combining the last two statements, we have that for each $x$ in $M$ there is an $s$ in $S$ such that $(T-r) x=s x$. In particular, there are elements $s_{1}, \ldots, s_{n}$ in $S$ such that $(T-r) x_{i}=s_{i} x_{i}$ for $1 \leq i \leq n$. Clearly, there are finitely many $A_{k}$ 's whose direct sum $B$ contains all of the $s_{i}$ 's. Let $N$ be the (nonunitary) $B$ submodule of $M$ generated by $\left\{x_{1}, \ldots, x_{n}\right\}$. The restriction $W$ of $T-r$ to $N$ is a $B$-endomorphism that leaves invariant every $B$-submodule. However, $B$ is strictly scalar-reflexive, since $B$ is a finite direct sum of strictly scalar-reflexive rings. Thus there is a $b$ in $B$ such that $W x=b x$ for every $x$ in $N$. Let $a=r e+b$. Then $T x_{i}=a x_{i}$ for $1 \leq i \leq n$. Thus $T x=a x$ for every $x$ in $M$; whence, $A$ is strictly scalar-reflexive.

The following lemma, which provides a class of rings that are not strictly scalarreflexive, is central to our later results on scalar-reflexive algebras over fields.

LEMMA 7. Suppose $S$ is a commutative ring, $a$ is a nonzero nilpotent in $S$, and $S / a S$ is a field. Then $R=S[y] /\left\langle y^{2}\right.$,ay is not strictly scalar-reflexive. In particular, if $F$ is a field, then $F[x, y] /\left\langle y^{2}, x^{n}, x y\right\rangle$ is not strictly scalar-reflexive for $n \geq 2$.

PROOF. Since the class of strictly scalar-reflexive rings is closed under homomorphic images, we can assume that $a^{2}=0$ (i.e., consider $R / a^{2} R$ ). Then $M=S \oplus S$ becomes an $R$-module with each $s$ in $S$ acting with the usual module multiplication and $y$ acting as the matrix $\left(\begin{array}{ll}0 & a \\ 0 & 0\end{array}\right)$. Let $T \in\left(\begin{array}{ll}a & 0 \\ 0 & 0\end{array}\right)$. Clearly, $T=\operatorname{End}_{R}(M)$ and $T$ is not a scalar. If $(u, v) \in M$, then $T(u, v)=(a u, 0)$. If $a v=0$, then $T(u, v)=a(u, v)$. If $a v \neq 0$, then $v$ must be invertible since the Jacobson radical $a S$ of $S$ does not contain $v\left(a^{2}=0\right)$ and $S / a S$ is a field. Thus $T(u, v)=u v^{-1} y(u, v)$. Thus $M$ is a finitely (doubly) generated $R$-module that is not scalar-reflexive, which implies $R$ is not strictly scalar-reflexive.

REMARK 8. Suppose $F$ is a field. It follows from Lemma 7 that $F[x, y]$ is not strictly scalar-reflexive. However $F[x]$ is strictly scalar-reflexive, $F(x, y)$ is strongly scalar-reflexive, and $F[x][y]=F[x, y] \subset F(x, y)$. Hence strict scalar-reflexivity is not preserved in subrings or in the formation of polynomial rings.

The next theorem is our main result. Most of the rest of this paper is devoted to proving a version of the Wedderburn principal theorem that will allow us to apply the following theorem. Throughout, $\mathscr{J}(R)$ denotes the Jacobson radical of a ring $R$.

THEOREM 9. Suppose that $A$ is a commutative algebra with identity over a field $F$ such that:

(a) $\mathscr{J}(A)$ is nilpotent,

(b) $A=F \cdot 1+\mathscr{J}(A)$.

Then the following are equivalent:

(1) $A$ is strongly scalar-reflexive,

(2) $A$ is strictly scalar-reflexive, 
(3) $A$ is an FGC ring,

(4) $A$ is isomorphic to $F[x] /\left\langle x^{n}\right\rangle$ for some positive integer $n$,

(5) $A$ is a PIR (principal ideal ring).

PROOF. The implications $(1) \Rightarrow(2)$ and $(4) \Rightarrow(5)$ are obvious. The implication $(4) \Rightarrow(1)$ is in Proposition 3 , the implication $(4) \Rightarrow(3)$ is in [8], and the implication $(3) \Rightarrow(2)$ is Proposition 4 .

$(2) \Rightarrow(4)$. Suppose that $A$ is strictly scalar-reflexive. We proceed by induction on the order $m$ of nilpotence of $\mathscr{J}(A)$. If $m=1$, then $A=F$ and (4) is true with $n=1$. Assume that $m>1$ and that the desired implication holds whenever the order of nilpotence is less than $m$. It follows that there is an element $a$ in $A$ such that $0 \neq a+\mathscr{J}(A)^{m-1} \in \mathscr{J}\left(A / \mathscr{J}(A)^{m-1}\right)$ and $A / \mathscr{J}(A)^{m-1}$ is generated by $1+\mathscr{J}(A)^{m-1}$ and $a+\mathscr{J}(A)^{m-1}$. Since $a^{m-1} \in \mathscr{J}(A)^{m-1}$, it follows that $a \in \mathscr{J}(A)$ and $a^{m}=0$. Also $A$ is generated by $\mathscr{J}(A)^{m-1}$ and the algebra $A_{0}$ is generated by 1 and $a$. We will show that $A=A_{0}$. Assume via contradiction that $b \in \mathscr{J}(A)^{m-1} \backslash A_{0}$. Choose a vector subspace (over $\left.F\right) \mathscr{J}$ of $\mathscr{J}(A)^{m-1}$ whose direct sum with the subspace spanned by $b$ and $A_{0} \cap \mathscr{J}(A)^{m-1}$ is $\mathscr{J}(A)^{m-1}$. Clearly, $\mathcal{J}$ is an ideal in $A$ and $A / \mathcal{J}$ is isomorphic to $F[x, y] /\left\langle x^{m}, y^{2}, x y\right\rangle$, which is not strictly scalar-reflexive (Lemma 7 ). This contradicts the fact that $A$ is strictly scalar-reflexive, which implies that $\mathscr{J}(A)^{m-1} \subset A_{0}$. Thus $A=A_{0}$.

$(5) \Rightarrow(4)$. If $A$ is a PIR, then there is a $y$ in $\mathscr{J}(A)$ such that $\mathscr{J}(A)=y A$. Thus $A=F+y A=F+y(F+y A)=F+F y+y^{2} A=\cdots=F[y]$, since $y$ is nilpotent. Thus (4) holds.

In order to apply Theorem 9 we need to prove a suitable version of the Wedderburn principal theorem. Let us say that a ring $R$ is a Wedderburn principal ring if there is a semisimple subring $S$ of $R$ such that $R=S+\mathscr{J}(R)$ (the additive direct sum). The following lemma is a consequence of the classical Wedderburn principal theorem [5]; we think that our proof may be more transparent than the usual ones.

LEMMA 10. Suppose that $F$ is a field, $f$ is an irreducible polynomial, and $n$ is a postive integer, $n \geq 2$. Then $R=F[x] /\left\langle f^{n}\right\rangle$ is a Wedderburn principal ring if and only if $f$ and its formal derivative $f^{\prime}$ are relatively prime.

ProOF. Suppose that $f$ and $f^{\prime}$ are relatively prime. Let $C$ be the companion matrix for the polynomial $f$. If $T$ is the $n \times n$ matrix (over $F[C]$ )

$$
\left(\begin{array}{cccccc}
C & I & 0 & \cdots & 0 & 0 \\
0 & C & I & \cdots & 0 & 0 \\
& & & & & \\
0 & 0 & 0 & \cdots & C & I \\
0 & 0 & 0 & \cdots & 0 & C
\end{array}\right),
$$

then, for each polynomial $q$ in $F[x], q(T)$ is an $n \times n$ upper triangular matrix with $q(C)$ as each entry on the main diagonal and $q^{\prime}(C)$ as each entry on the first diagonal above the main diagonal. Furthermore, since $f(C)=0$ and the polynomials $f$ and $f^{\prime}$ are relatively prime, it follows that $f^{\prime}(C)$ is invertible, and that the inverse of $f^{\prime}(C)$ is a polynomial $q(C)$ in $C$. Thus the minimal polynomial of $T$ is $f^{n}$, and the ring $R$ is isomorphic to the ring of polynomials in $T$. Since $\mathcal{J}(R)=f R$, and $R / f R$ is isomorphic to $F[x] /\langle f\rangle$, it suffices to show that $R$ contains an element $a$ with $f(a)=0$. The principal of mathematical induction implies that we must 
show that if $n>1$, then $f(b)^{m}=0$ for some $b$ in $R$ and some positive integer $m<n$. Let $J$ be the $n \times n$ matrix obtained by replacing all of the $C$ 's with 0 's in the matrix for $T$. A matrix computation shows that $q(T) f(T)$ is the sum of $J$ and a multiple of $J^{2}$. Thus $f(T-q(T) f(T))$ is a multiple of $J^{2}$, from which it follows that $f(T-q(T) f(T))^{m}=0$ for some positive integer $m, m<n$. Thus $R$ is a Wedderburn principal ring.

Conversely, suppose that $f$ and $f^{\prime}$ are not relatively prime. Then $p=\operatorname{char}(F) \neq$ 0 , and $f^{\prime}=0$. Thus $f=g^{p}-w$ for some $w$ in $F$. Assume via contradiction that $R$ is a Wedderburn principal ring. Then there is a polynomial $h$ such that $f(h)=0$ in $R$, i.e., $f^{n}$ divides $f(h)$. Thus $(g(h)-g)^{p}=f(h)-f$ is a multiple of $f$. Since $f$ is irreducible, $f$ divides $g(h)-g$, and therefore $f^{p}$ divides $(g(h)-g)^{p}$. Thus $f=f(h)-(g(h)-g)^{p}$ is a multiple of $f^{2}$, since $f^{p}$ divides $(g(h)-g)^{p}$ and $f^{n}$ divides $f(h)$. This contradiction completes the proof.

Suppose that $K$ is an extension field of a field $F$. We say that $K$ is a simple elementary extension of $F$ if $K$ is either a simple transcendental extension of $F$ or $K$ is a simple extension by an element whose minimal polynomial has the form $f^{n}$ with $f$ an irreducible polynomial such that $f$ and $f^{\prime}$ are relatively prime. An elementary chain from $F$ to $K$ is a chain $\left\{F_{\sigma}: \sigma \leq \beta\right\}$ of fields ( $\beta$ is an ordinal number) such that $F_{0}=F, F_{\beta}=K, F_{\sigma+1}$ is a simple elementary extension for each $\sigma<\beta$, and for each limit ordinal $\sigma$, the field $F_{\sigma}$ is the union of the $F_{\delta}$ 's $(\delta<\sigma)$. We say that the elementary chain $\left\{F_{\sigma}\right\}$ is purely transcendental (resp., purely algebraic) if each $F_{\sigma+1}$ is a transcendental (resp., algebraic) extension of $F_{\sigma}$. We say that $K$ is an elementary extension of $F$ if there is an elementary chain from $F$ to $K$; if the chain is purely algebraic (resp., purely transcendental), we say that $K$ is a purely algebraic (resp., purely transcendental) elementary extension of $F$. Note that if $K$ is a separable algebraic extension of $F$, then $K$ is a purely algebraic elementary extension of $F$, and if $\operatorname{char}(F)=0$, then every extension of $F$ is elementary. Also note that in (2) and (3) below the assumption that $\mathscr{J}(R)$ is nil can be replaced by the weaker assumption that $R$ is an SBI ring, i.e., for each $y$ in $\mathscr{J}(R)$, there is an $x$ in $R$ such that $x^{2}+x=y$ (see [7, pp. 124-126]).

PROPOSITION 11. Suppose that $R$ is a commutative ring with identity.

(1) If $R / \mathscr{J}(R)$ is a field that is a purely transcendental extension of $Q$, then $R$ is a Wedderburn principal ring.

(2) If $\mathscr{J}(R)$ is nil, $R$ is an algebra over a field $F$, and $R / \mathscr{J}(R)$ is a finite direct sum of fields that are elementary extensions of $F$, then $R$ is a Wedderburn principal ring.

(3) If $\mathcal{J}(R)$ is nil and $R / \mathcal{J}(R)$ is a finite direct sum of fields of characteristic 0 , then $R$ is a Wedderburn principal ring.

PROOF. (2). Let $\tau: R \rightarrow R / \mathscr{J}(R)$ be the quotient map. If $a \in R$ and $\tau(a)$ is an idempotent, then $a(1-a)$ is nilpotent, since $\mathscr{J}(R)$ is nil. It therefore follows (see [4, Lemma 1.3.21]) that there is an idempotent $p$ in $R$ such that $\tau(a)=\tau(p)$. But $R / \mathscr{J}(R)$ is a finite direct sum of fields; thus $R$ is a finite direct sum of rings with nil Jacobson radical whose quotient by the radical is a field. Hence we can assume that $R / \mathscr{J}(R)$ is a field. By hypoothesis, there is an elementary chain $\left\{F_{\sigma}: \sigma \leq \beta\right\}$ from $F$ to $R / \mathcal{J}(R)$. Using Zorn's lemma we can choose a maximal (with respect to inclusion) subfield $E$ of $R$ such that $\tau(E)=F_{\sigma}$ for some $\sigma$. Assume 
via contradiction that $\sigma \neq \beta$. Then there is an element $b$ in $F_{\sigma+1}$ such that $F_{\sigma+1}=F_{\sigma}(b)$. First suppose that $b$ is algebraic over $F_{\sigma}$, with a minimal polynomial $f^{n}$ with $f$ irreducible and $f$ and $f^{\prime}$ are relatively prime. Choose $a$ in $R$ so that $\tau(a)=b$. Then $\tau(f(a))=f(\tau(a))=0$ (identifying $E$ with $F_{\sigma}$ ), which implies that $E[a]$ is isomorphic to $E[x] /\left\langle f^{n}\right\rangle$ for some positive integer $n$. Thus, by Lemma 10, we know that $E[a]$ contains a field $E^{\prime}$ such that $\tau\left(E^{\prime}\right)=F_{\sigma+1}$, which violates the maximality of $E$. This contradiction implies that $b$ must be transcendental over $F_{\sigma}$. However, even without the assumption that $\mathscr{J}(R)$ is nil, we have for each $c$ in $R$, that $c$ is invertible if and only if $\tau(c)$ is invertible. (Proof: If $\tau(c) \tau(d)=1=\tau(1)$, then $1-c d \in \mathscr{J}(A)$, which implies that $c d=1-(1-c d)$ is invertible. Since $c d=d c$, it follows that $c$ is (both left and right) invertible.) It follows that if $g$ is a nonzero polynomial in $E[x]$, then $g(a)$ is invertible. Thus $\{g(a) / h(a): g, h \in E[x], h \neq 0\}$ is a field whose image under $\tau$ is $F_{\sigma+1}$, a contradiction implying that $\sigma=\beta$. Hence $\tau(E)=R / \mathcal{J}(R)$, which implies that $R$ is a Wedderburn principal ring.

(1) Since $R / \mathscr{J}(R)$ is a field with characteristic 0 , it follows that each nonzero integer multiple of the identity is invertible. Hence $R$ is an algebra over $Q$, and the desired conclusion follows from imitating the proof of (2).

(3) As in the proof of (2), we can assume that $R / \mathscr{J}(R)$ is a field (with characteristic 0 ), and as in the proof of (1), $R$ is an algebra over $Q$. Since there is an elementary chain from $Q$ to $R / \mathscr{J}(R)(\operatorname{char}(Q)=0)$, the desired conclusion follows from (2).

We conclude with the promised application of Theorem 9 .

THEOREM 12. Suppose that $R$ is a commutative Wedderburn principal ring with identity such that $\mathcal{J}(R)$ is nilpotent and $R / \mathcal{J}(R)$ is a finite direct sum of fields. The following are equivalent.

(1) $R$ is strongly scalar-reflexive,

(2) $R$ is strictly scalar-reflexive,

(3) $R$ is an FGC ring,

(4) $R$ is a finite direct sum of rings of the form $F[x] /\left\langle x^{n}\right\rangle$, with $F$ a field and $n$ a positive integer,

(5) $R$ is a PIR.

PROOF. It follows that $R$ is a finite direct sum of rings $R_{k}$ such that each $R_{k}$ is an algebra over a field $F_{k}, R_{k}=F_{k}+\mathscr{J}\left(R_{k}\right)$. The proof now follows from Theorem 9.

In summary we see that the classes of strongly and strictly scalar-reflexive rings arise from very natural algebraic questions. The class of strongly scalar-reflexive rings is closed under finite direct sums and quotients, and it contains the rings that are nontrivial quotients of PID's.

The class of strictly scalar-reflexive rings is closed under more general direct sums, and the general structure of such rings seems much more complicated. There are many interesting questions that one can ask about this class, e.g., whether it is closed under direct products or localizations. We feel that these and other questions about scalar-reflexivity deserve serious study.

The first author wishes to thank the National Science Foundation for its support while this research was undertaken. 


\section{REFERENCES}

1. E. Azoff, K-reflexivity in finite-dimensional spaces, Duke Math. J. 40 (1973), 821-830.

2. L. Brickman and P. A. Fillmore, The invariant subspace lattice of a linear transformation, Canad. J. Math. 19 (1967), 810-822.

3. D. W. Hadwin, Algebraically reflexive linear transformations, Linear and Multilinear Alg. 14 (1983), 139-147.

4. I. N. Herstein, Noncommutative rings, Carus Math. Monographs, No. 15, Math. Assoc. of Amer., 1973.

5. N. Jacobson, Basic algebra. II, Freeman, San Francisco, Calif., 1980.

6. I. Kaplansky, Infinite abelian groups, Univ. of Michigan Press, Ann Arbor, 1954.

7. __ Fields and rings, Univ. of Chicago Press, Chicago, 1972.

8. R. Wiegand and S. Wiegand, Commutative rings whose finitely generated modules are direct sums of cyclics, Abelian Group Theory (Proc. Second New Mexico State Univ. Conf., Las Cruces, N.M.), Lecture Notes in Math., vol. 616, Springer-Verlag, Berlin, 1977, pp. 406-423.

Department of Mathematics, UNiversity of NeW Hampshire, Durham, NeW HAMPSHIRE 03824

Department of Mathematics, Michigan State University, East Lansing, MICHIGAN 48824 